\title{
DERECHO E INTERNET •
}

\section{FERNANDO GALINDO*}

\begin{abstract}
Resumen
Junto con otros profesores iniciamos varias investigaciones interdisciplinares dirigidas a establecer relaciones entre derecho e informática. El tema central de las investigaciones era la comunicación jurídica, y más en concreto, el estudio de las posibilidades de poner a disposición de juristas, por medio de tesauros, el almacenamiento y la recuperación de documentación jurídica, utilizando como instrumento auxiliar las Tecnologías de la Información y la Comunicación. En cuanto Internet se hizo real apareció otro tipo de investigación: se consideró cuáles eran los requisitos a satisfacer para garantizar los derechos de las personas a comunicar libremente sus pensamientos en la recuperación o acceso a la información. Estos trabajos fueron los dedicados a gestión de la identificación y firma electrónica. En la actualidad volvemos a la idea de tesauro combinándola con la de firma, intentando poner en práctica el objetivo de construir sistemas que permitan, utilizando internet en la mayor medida posible, a todos los ciudadanos en la sociedad en red, el libre acceso y recuperación de documentación jurídica. Labor que es consecuencia de las virtualidades de internet: es posible realizar trabajos conjuntos, de carácter "colaborativo", entre grupos de investigación dedicados a derecho e informática que permitan dicho amplio y, a la vez, concreto acceso. Este fue el punto de partida para establecer la Red Jurídica para la Sociedad de la Información: LEFIS (Legal Framework for the Information Society), organización fundada por el grupo de investigación.
\end{abstract}

Palabras clave.- Derecho e informática - Sociedad en red - Tecnologías de la información y la comunicación - Red LEFIS.

\begin{abstract}
Jointly with other professors, we began several interdisciplinary investigations aimed at establishing relations between the law and information technology. The central subject of the investigations was the legal communication, and more specifically, the study of the possibility of providing scholars, through thesaurus, with the storage and recovery of legal documentation, using the information and communication technologies as an auxiliary instrument. As soon as the Internet was materialized, another type of research appeared, considering the requirements to be met to guarantee the rights of persons to freely communicate their thoughts in the recovery of or access to the information. The referred works were those dedicated to the management of electronic identification and signature. At present, we return to the idea of the thesaurus combining it with the idea of the signature, in an attempt to put into practice the goal of building a system that will allow - making use of the Internet as much as possible - all citizens in the online society, free access
\end{abstract}

\footnotetext{
* Departamento de Derecho Penal, Filosofía del Derecho e Historia del Derecho. Universidad de Zaragoza. cfa@unizar.es

- El presente trabajo es el texto de la ponencia que con el mismo título fue presentada en el XIII Congreso de FIADI que tuvo lugar en la Universidad de Lima entre el 10 y el 14 de noviembre de 2009.
} 


\begin{abstract}
to and the recovery of legal documentation. A task that is a consequence of the virtual qualities of the Internet: joint works, of a "collaborative" nature can be carried out among research groups engaged in law and information technology that will enable the referred wide and, at the same time, specific access. This was the starting point to establish the Legal Framework for the Information Society or LEFIS, an organization founded by the research group.
\end{abstract}

Key words: Law and Information Technology - online society - Information and Communication Technologies - LEFIS network.

\title{
Sumario
}

1. Introducción. 2. Tesaurus. 3. Identificación. 4. La sociedad en red. 4.1. Materias. 4.2. El campus virtual compartido en derecho y tecnologías de la información y la comunicación. 4.3. La red LEFIS. 5.Conclusión.

\section{INTRODUCCIÓN}

El presente trabajo quiere introducir a la relación entre derecho e internet a partir de la experiencia desarrollada en derecho e informática, contando con determinados datos: el cambio del objeto de interés que ha acarreado la aparición de internet para un conjunto de investigaciones que fueron iniciados en los años ochenta del pasado siglo y que se mantienen hasta la actualidad. El procedimiento va a permitir expresar algunas de las implicaciones que comporta el uso de internet en el terreno del derecho y la informática.

El trabajo quiere dar cuenta, en concreto, de varias investigaciones, realizadas desde los años ochenta hasta la actualidad, caracterizadas por el hecho de que, tomando como objetivo guía de las mismas la elaboración de propuestas orientadas a promover el cambio de las prácticas jurídicas en sociedades democráticas, han utilizado como instrumento para construirlas los recursos tecnológicos propios del momento histórico estudiado, una vez que el desarrollo tecnológico es uno de los causantes de los cambios sufridos por las actividades de los profesionales del derecho a lo largo del periodo, así como ha posibilitado el establecimiento y consolidación de la "sociedad en red"1 en la que vivimos.

Las referencias tecnológicas concretas utilizadas han sido:

1. Las virtualidades de los sistemas de recuperación de documentación, que permitieron ampliar y expandir el acceso del contenido de las normas jurídicas a los ciudadanos, desde mediados de los ochenta, facilitando su salida del círculo limitado del conocimiento de las mismas por los espe- 
cialistas en derecho, colaborando así a posibilitar la puesta en acción del principio democrático que establece que la ley es la voluntad del pueblo, creada por sus representantes en asamblea en nombre del pueblo².

2. El uso de las técnicas criptográficas como instrumentos de garantía democrática de la expansión de internet, al constituirse las mismas como medio de preservar la puesta en práctica de los principios jurídicos fundamentales del Estado de derecho, especialmente los referidos a la salvaguarda de las libertades de expresión y opinión, desde mediados de los noventa, en relación al uso de las tecnologías de la información y la comunicación (TIC) en la transmisión y recepción de comunicaciones ${ }^{3}$, y

3. El uso democrático de las TIC en la práctica jurídica profesional diaria como instrumentos inevitables para la puesta en realidad de la "sociedad en red", en relación a la procuración de servicios públicos por parte de las administraciones e impartición de justicia a través de los procedimientos judiciales propios del Estado de derecho, que se prevé tengan lugar en la actualidad y próximos años (Saarenpää, 2008).

De todo ello trata este trabajo. Con lo cual nos ocupamos aquí de presentar, resumidamente, las actividades principales realizadas por el grupo interdisciplinario de investigación denominado "Protección de datos y firma electrónica" ${ }^{4}$, en relación a los siguientes objetos de investigación:

1. Tesauros jurídicos.

2. Uso de mecanismos de identificación de personas.

3. Establecimiento democrático de la sociedad en red con respecto al ejercicio de actividades jurídicas por profesionales del derecho, y

4. Constitución, implantación y mantenimiento de la red LEFIS 5 junto con otros grupos de investigación, como forma de hacer operativa en la "sociedad en red" la relación entre derecho e internet en el ámbito del derecho y la informática.

Cada apartado de este trabajo (del 2 al 4) se dedica a la presentación de las investigaciones. El último apartado (5) propone una conclusión.

\section{TESAUROS}

En la segunda mitad de los ochenta del siglo $X X$, cuando se trataba de explorar las posibilidades que ofrecían las "Tecnologías de la información y la comunicación (TIC) al ámbito jurídico, iniciada la aplicación de dichas TIC 
a la gestión de la administración de justicia en juzgados y tribunales, parecía adecuado, a efectos de considerar posibles consecuencias de dicha aplicación en la actividades jurídicas propias del Estado de derecho y despertar interés sobre la materia a futuros juristas formados en las facultades de derecho, tomar como referencia para la exploración de dichas posibilidades a teorías del derecho que preconizaban realizar un estudio del derecho a partir de las características propias del lenguaje de las normas en cuanto expresión formal de las leyes aprobadas en el Parlamento por los representantes democráticos de los ciudadanos.

Existía en la época un amplio consenso doctrinal que estimaba que teorías y técnicas TIC deberían auxiliar a la generación automática de normas (SánchezMazas, 1978) (DeBessonet, 1984).

El establecimiento de colecciones de palabras, convenientemente estudiadas y depuradas, expresivas de textos jurídicos o problemas jurídicos, recopiladas en forma de diccionarios o tesauros era la primera fase del trabajo a realizar, que estaba destinado a la generación posterior de normas.

Ello era todavía más factible si ocurría, como así se quería hacer en las investigaciones emprendidas por el grupo, que se consideraban variantes de dichas teorías del derecho las posibilidades que ofrecían aspectos concretos de la discusión filosófico-jurídica del momento. Especialmente los estudios realizados desde la teoría de la argumentación jurídica sobre las diferentes actividades propias de los profesionales del derecho en el Estado de derecho: interpretación y aplicación de las leyes fundamentales (Aarnio, Alexy, Peczenick, 1981) (Habermas, 1981).

A estos efectos, conjuntamente con varios profesores procedentes de las matemáticas (algebra y estadística), y de las materias dogmáticas que se explican en las facultades de derecho desde el siglo XIX (en concreto derecho civil, derecho penal y derecho administrativo), comenzamos (desde la filosofía del derecho), en la época señalada (mediados de los ochenta), a realizar investigaciones interdisciplinares relacionadas, en el marco teórico señalado, con los resultados más provechosos para la época de las TIC: sus posibilidades para almacenar y recuperar documentación jurídica, y para representar el conocimiento jurídico por medio de los lenguajes de programación lógica.

Con esto se pone de manifiesto que el objetivo de las investigaciones iba más allá de la recuperación de documentación. Se quería, también, utilizar las posibilidades que ofrecían los programas denominados "sistemas expertos" 
para acceder y recuperar textos jurídicos de interés local. El ejemplo inicial quedó constituido por la legislación de carácter civil propia de la Comunidad Autónoma de Aragón en España.

Con ello se quería poner a disposición de expertos jurídicos y ciudadanos dicha normativa utilizando como herramienta auxiliar diálogos y argumentos típicos en los que quedaban esquematizadas o representadas las actividades jurídicas.

A esta investigación la denominamos construcción de un tesauro jurídico inteligente ${ }^{6}$.

Las consecuencias de estas actividades fueron excelentes en lo que se refiere a la construcción de bases de datos jurídicas integradas por legislación, jurisprudencia y doctrina sobre una materia de especial interés en la Comunidad Autónoma como el derecho histórico y vigente de Aragón 7 . En efecto, fruto de las investigaciones fue el establecimiento de canales, las expresadas bases de datos, que permitían acceder a juristas y ciudadanos interesados el contenido de un derecho cuya vida fue limitada por razones históricas: la tendencia centralizadora que se asentó en España a partir del XVIII por influencia del racionalismo ilustrado propio de la organización del poder político de la época, tendencia rectificada a partir del establecimiento de la Constitución española de 1978, básicamente, reconocedora del Estado de las Autonomías y del ejercicio del principio político de separación de poderes por las entidades autonómicas.

Distinto fue el resultado de las investigaciones en lo relativo a la construcción de sistemas expertos, que facilitaran a ciudadanos y especialistas el acceso a textos jurídicos concretos referidos al ejercicio de derechos propios facultados por el ordenamiento. Estos sistemas, efectivamente, se construyeron pero no pudieron pasar de la fase de prototipo $^{8}$, siendo expresados en forma de programas construidos en lenguajes de programación propios de la denominada inteligencia artificial.

Los sistemas expertos eran programas que respondían a través de diálogos a posibles preguntas simples referidas a cuáles son algunas de las normas específicas, distintas a las generales españolas, que regulaban la obtención de la mayoría de edad en la Comunidad Autónoma de Aragón.

Más adelante se desarrollaron por el grupo otras investigaciones que representaban no tanto el contenido de normas cuanto que el de acciones procesa- 
les a plantear en un juzgado o tribunal sobre determinada materia ${ }^{9}$. Con ello se incrementó la eficacia del modelo pero este seguía sin poder probar su utilidad al faltar los recursos e interés con los que pasar a la prueba "industrial" del mismo.

La conclusión alcanzada en estas investigaciones estaba referida a que su planteamiento era preliminar, hablando en términos tecnológicos, y a que no existía interés por la aplicación de las mismas. En aquellos tiempos comenzaba la lenta explotación de la documentación jurídica que quedaba almacenada en soporte digital por empresas e instituciones públicas. Se observó que en la época no era posible obtener, al menos en el contexto español, resultados prácticos, programas o aplicaciones originadas a partir de este tipo de investigación.

Hay que decir que en cambio, sí que se obtuvo como producto de las investigaciones referidas detallados estudios y aproximaciones referidas a la expresión de las características propias de la actividad del acceso a textos jurídicos, y del resto de actividades profesionales de juristas ${ }^{10}$, así como al concepto de derecho, llegando a definir a este como actividad justa de juristas con relación a textos jurídicos (Galindo, 1993) ${ }^{11}$.

\section{IDENTIFICACIÓN}

En cuanto la red internet se hizo operativa, comenzando a ponerse en acción las comunicaciones en tiempo real por medio del uso de las TIC, al, inicialmente, poderse enviar y recibir mensajes a través del correo electrónico, o comprar productos a proveedores de bienes y servicios a través de internet, mediante lo que muy pronto se denominó comercio electrónico, se consideraron como objetos de investigación propios del grupo, los siguientes:

1. La continuación del desarrollo de propuestas dirigidas a auxiliar a las actividades jurídicas utilizando sistemas de recuperación de información, auxiliados por las telecomunicaciones, más sofisticados, en relación a dominios concretos $^{12}$.

2. La elaboración de propuestas dirigidas a garantizar los derechos de personas concretas a comunicar sus pensamientos y decisiones libremente en el momento de transmitir y recibir información, utilizando recursos que posibilitaban una mayor y mejor recuperación de información jurídica, en forma adaptada a necesidades concretas de usuarios identificables. Estos fueron los trabajos dirigidos a garantizar la gestión de la identificación de 
emisores y receptores de mensajes y la garantía de la preservación de la integridad del contenido de los mensajes por medio de la firma electrónica ${ }^{13}$.

Estas investigaciones han sido desarrolladas desde la segunda mitad de los noventa.

Las investigaciones sobre elaboración de sistemas de recuperación de documentación jurídica estuvieron influidas por el hecho de que en España el acceso libre a la documentación jurídica por todos los ciudadanos se produjo por iniciativa de las instituciones públicas responsables de su creación, paulatinamente, desde la segunda mitad de los años noventa. En otros países no tuvieron lugar estas iniciativas públicas. Esto hizo que en España no surgiera la necesidad del establecimiento de centros de investigación que se ocuparan de hacer públicos, de forma no oficial, los textos jurídicos utilizando las TIC.

Se entendía fácilmente el surgimiento de dichos centros de investigación. Según los principios del Estado de derecho las normas son de acceso público: las elaboran los representantes de los ciudadanos. Si solo eran accesibles en su literalidad mediante la utilización de sistemas de recuperación de documentación cuyos propietarios eran empresas, cuyos servicios debían ser pagados por sus usuarios, no se satisfacían los principios de libre acceso a los textos jurídicos. Los centros de investigación de otros países se ocupaban, por ello, de romper esta dinámica de comercialización de la difusión de los textos jurídicos almacenados en formato digital, promoviendo su publicidad mediante el establecimiento de colecciones documentales "no oficiales" "on line" abiertas al acceso de las mismas por todos los ciudadanos ${ }^{14}$.

En cambio en España, como decimos, la legislación y los reglamentos administrativos se han hecho accesibles por los canales oficiales a cualquier persona, libremente, en internet desde $1995^{15}$. Desde esas fechas el Boletin Oficial del Estado, órgano oficial de publicación de las normas estatales está a disposición de los ciudadanos. Lo mismo ocurre, prácticamente, con respecto a las normas promulgadas por todas las comunidades autónomas.

Estas iniciativas se generalizaron formalmente a partir de 1 de enero de 2004 .

En lo referido a la documentación judicial, en 1997 el Consejo General del Poder Judicial estableció el Centro de Documentación Judicial como entidad responsable para hacer públicas las sentencias del Tribunal Supremo, los Tribunales Superiores de Justicia de las Comunidades Autónomas, Audiencias y otras entidades judiciales. 
El campo de acción de las empresas quedaba abierto, en uno y otro caso, en el sentido de que ellas, siguiendo las pautas establecidas para el tratamiento y recuperación de documentación jurídica por el Consejo de Europa, la Comunidad Europea y las normas propias de cada país, podían dar acceso a dicha documentación utilizando sistemas de recuperación desarrollados por las mismas, percibiendo a cambio de sus servicios el importe correspondiente.

Estas circunstancias originaron que el grupo de investigación centrara su trabajo, no tanto en la construcción de sistemas genéricos de recuperación de documentación jurídica, cuanto que en la construcción de sistemas de ayuda a las decisiones jurídicas hechas por instituciones concretas, o por la propia administración, los juzgados y los profesionales del derecho, utilizando nuevos sistemas TIC, como el correo electrónico e internet, cuyo uso comenzaba a generalizarse en la época considerada (segunda mitad de los noventa) ${ }^{16}$.

La estrategia permitió fijarse en el estudio de los cambios organizativos y funcionales que estos usos reclamaban de las diferentes organizaciones y agentes jurídicos. Es por ello que a comienzos de los años dos mil el estudio se centró en lo que se iniciaba en la época nominándolo con la expresión gobierno electrónico o administración electrónica, pudiendo estudiarse así la expansión del uso de las técnicas de gobernanza, o de eficacia, en la acción jurídica de las administraciones públicas ${ }^{17}$.

A la vez se puso especial énfasis en el estudio de las características y requisitos de la transmisión y recepción de mensajes electrónicos utilizando internet. Especialmente se estudiaron sus virtualidades (las posibilidades de comunicarse en tiempo real con interlocutores situados en cualquier lugar del mundo), sus debilidades (las posibilidades de conocerse por terceros el contenido de los mensajes y la suplantación de emisores o receptores de mensajes) y la solución a estas últimas. Solución que, desde la segunda mitad de los noventa, quedó centrada, especialmente, también normativamente, en el uso del cifrado de clave pública, al considerarlo como un recurso adecuado, capaz de solventar las debilidades de los sistemas de comunicaciones facilitados por internet consistentes en el hecho de que las comunicaciones realizadas con este recurso eran susceptibles de ser vistas, modificadas y falsificadas por terceras personas.

Era el momento de la promulgación de directivas europeas y normas estatales centradas en la regulación de la "firma electrónica", la construcción de infraestructuras de clave pública y la regulación de los servicios de la sociedad de la información, que permitían hacer realidad dicha normativa ${ }^{18}$. 
De aquellos años (más precisamente: de 1997 a 2000) son los trabajos del grupo destinados a discurrir, junto a juristas como notarios, registradores de la propiedad, procuradores de los tribunales y abogados, sobre las características que debía tener la confianza en el uso de las TIC cuando se recurría a internet, y a proponer la construcción de instituciones o sistemas, entidades de certificación de clave pública, dedicados a potenciarla y promoverla atendiendo a los principios propios del Estado de derecho ${ }^{19}$.

Se hablaba de confianza porque la utilización de las claves públicas como recurso para establecer la solución a las debilidades de las comunicaciones de internet mediante el uso de su cifrado, requería el establecimiento de servicios de infraestructura de clave pública, que tenían como función asegurar que la clave identificativa de los mismos usuarios o de los mensajes enviados cifrados era propia de una u otra persona que había confiado sus datos personales como titular de la clave a una tercera parte: la entidad titular de la infraestructura de clave pública. La confianza requería depositar información personal en terceras partes, con el fin de lograr una identificación segura en internet utilizando claves públicas, o lo que es lo mismo en la práctica del comercio hecha utilizando los medios de comunicación (las TIC) ${ }^{20} \mathrm{o}$, más recientemente, en el acceso a los servicios públicos a través de lo que se ha venido a denominar administración electrónica o gobierno electrónico.

En esta época el grupo constituyó entidades de certificación de clave pública junto a notarios ${ }^{21}$ y registradores, que sirvieron de base para el establecimiento de entidades de certificación no oficiales administradas por el grupo de investigación, que son utilizadas en la actualidad en ámbitos docentes y de investigación ${ }^{22}$.

\section{LA SOCIEDAD EN RED}

Por el desarrollo tecnológico sucedido en los últimos años, a nadie le puede extrañar que en la actualidad sea posible el funcionamiento de la sociedad en red, también denominada sociedad omnipresente ${ }^{23}$. Es decir, el funcionamiento de una sociedad en la que se puede realizar actividades concretas, con obtención instantánea de efectos jurídicos y económicos para las mismas, desde cualquier lugar (el de residencia habitual u otro), siempre que se cuente con una conexión TIC.

Así es: la realización de actividades a distancia como compraventas de productos, obtención de servicios públicos (certificados, cobro de pensiones), satisfacción de obligaciones como personas y ciudadanos (pago de tributos), transacciones económicas, transacciones bancarias, trabajo desde casa o a dis- 
tancia, aprendizaje, visionado de películas, visionado de televisión, consulta de documentación, uso de medios audiovisuales, realización de juegos [...] son posibles, teniendo los correspondientes efectos, siempre que se cuente con herramientas TIC y mecanismos que permitan, especialmente, como hemos visto en el apartado anterior, la identificación fiable de quienes las realizan.

Por lo mismo, también es posible, utilizando idénticos recursos, la realización a distancia de actividades propias de profesionales del derecho: envío y recepción de notificaciones, transmisión de documentos, grabación en video y audio de procesos judiciales, realización de testificaciones, presentación de informes de peritos, interrogatorios a testigos y acusados, declaraciones de las partes en un proceso [...].

Ha de tenerse en cuenta, además, que ello es propiciado por el hecho de que desde una perspectiva política, vivimos hoy en la sociedad de la gobernanza, expresión en la que se resume la puesta en práctica por las instituciones públicas del ideal de eficiencia y sus reglas, ya probadas en el ámbito privado: en la gestión de empresas, en la gestión administrativa, y que estas reglas se van convirtiendo progresivamente en reglas de acción propias de la administración pública o de la administración de justicia, en convivencia con otros principios jurídicos de acción como son los de participación y legalidad propios del Estado de derecho ${ }^{24}$.

Todo lo cual significa que han venido a introducirse en el ámbito jurídico pautas de acción que no son las que lo regían hasta hace poco. Especialmente ha cobrado trascendencia el uso de las técnicas de gestión desarrolladas en el ámbito empresarial, a diferencia del pasado en el que la gestión de carácter administrativo o judicial estaba ordenada, básicamente, por la aplicación del principio de legalidad.

Los cambios están reconocidos institucionalmente: hay que tener en cuenta la existencia de normas referidas a cambios organizativos o procesales de la administración de justicia, por ejemplo, que han plasmado en la reforma de la oficina judicial. Estas normas distinguen funcional e institucionalmente entre la labor del juez, que toma decisiones judiciales propiamente dichas, y la del secretario o personal auxiliar, que se ocupan de la gestión de la tramitación de las mismas, o propugnan el establecimiento de cartas de servicios en dicha administración.

Es otro hecho, prescrito por la legislación, el uso de las TIC para agilizar las comunicaciones que tienen lugar entre los juzgados y, por supuesto, entre los ciudadanos y las oficinas responsables de los servicios públicos, justamente con el fin de mejorar la calidad de los servicios prestados a los ciudadanos. 
Estas circunstancias han hecho al grupo continuar, con mayor razón que la existente en los años ochenta, las líneas de investigación dirigidas a estudiar y comprender cuáles son las características de las actividades jurídicas democráticas en la sociedad en red, y a proponer a futuros juristas pautas de aprendizaje y reflexión críticas, coherentes con la dotación de las competencias profesionales que son precisas para realizar dichas actividades, con el fin de que puedan realizar conscientemente, reflexivamente, críticamente, democráticamente, su trabajo ${ }^{25}$. Trabajo que tiene características distintas al de tiempos recientes en los que, por ejemplo, la función de las TIC quedaba reducida a ser la de buenos instrumentos auxiliares o mejoradas máquinas de escribir, o programas que facilitaban, genéricamente, la recuperación de documentación jurídica.

Con ello se responde al hecho de que en la actualidad es posible que personas concretas ejerciten sus obligaciones y derechos como profesionales del derecho, trabajador, profesional, estudiante, propietario, vendedor, usuario, comprador o ciudadano desde una terminal TIC: ordenador o teléfono móvil, por ejemplo, a la vez que quedan preservadas las garantías que establecen los ordenamientos democráticos al ejercicio de los respectivos derechos y obligaciones.

Es por ello que en la actualidad el grupo continúa poniendo en práctica la idea de elaborar un "tesauro jurídico inteligente" que permita un acceso individualizado a documentación jurídica, o lo que es lo mismo continúa la construcción de sistemas de recuperación de documentación, junto a la de infraestructuras de clave pública que dan confianza al uso de la firma electrónica, intentando hacer real el objetivo de construir sistemas que permitan, en la mayor medida posible, a personas legitimadas, el libre acceso, la transmisión y la recuperación de documentación jurídica utilizando internet y poner en práctica el ejercicio de derechos y deberes que proporciona el derecho, usando su idioma propio, sea el que sea tanto el lugar físico en el que dicha documentación jurídica se encuentre almacenada, como el lugar desde el que la misma se quiera utilizar.

A los efectos indicados el grupo de investigación, junto a otros grupos, realiza aportaciones doctrinales dirigidas a entender esta realidad, a la vez que diseña, a modo de ejemplo, ofertas docentes, módulos, orientados a la ejercitación de profesionales del derecho y estudiantes (sean o no juristas) en las competencias y habilidades que les son requeridas por las características de las funciones jurídicas a desarrollar en el contexto del Estado de derecho, fomentando la democracia, la gobernanza y las potencialidades que ofrece la sociedad en red. 
Las aportaciones tienen un triple carácter:

1. Por un lado, hacen referencia al contenido de la docencia, que ha de tener propuestas de contenido adaptadas a las necesidades de funcionamiento de la organización de la sociedad democrática e instituciones jurídicas en red ${ }^{26}$,

2. Por otro, son primeros ensayos para el ejercicio de las actividades jurídicas en la sociedad en red, considerando la complejidad que conlleva una actuación profesional democrática en dicha sociedad, estando dirigida la experiencia del campus virtual sobre derecho y TIC que el grupo constituye para la satisfacción de este objetivo ${ }^{27}$, y;

3. Las aportaciones son colectivas y realizadas gracias a internet. En efecto: las actividades son posibles porque el grupo ha creado la red LEFIS (Legal Framework for the Information Society), "motor" internacional para investigación, desarrollo y docencia en relación a derecho e informática en la sociedad en red.

A todo ello nos referimos a continuación.

\subsection{Materias}

Las materias, módulos o contenidos docentes, son respuesta a elencos de competencias y habilidades para el jurista que han sido precisadas por el grupo de investigación, siguiendo las pautas de reforma docente propuestas por el proceso de cambio de la docencia universitaria europea (Espacio Europeo de Enseñanza Superior: EEES) denominado proceso de Bolonia, a través de varias iniciativas, en una lista de las mismas (competencias y habilidades) como la siguiente:

- Acceso a textos legales.

- Aplicación de los conocimientos a la práctica.

- Capacidad de razonar y argumentar jurídicamente.

- Comprensión e interpretación de las normas jurídicas y aplicación a la práctica.

- Conocimientos sobre derecho de las TIC.

- Interpretar las normas conforme a las circunstancias del caso y a la realidad social del momento en que deben ser aplicadas.

- Saber presentar información verbal y visualmente.

- Trabajar en equipo.

Las denominaciones de temas, materias o módulos docentes, a tratar para adquirir experiencia en competencias y habilidades profesionales se encuentran 
recogidas en la siguiente lista de cursos que constituye la oferta docente típica de los grupos de investigación que trabajan conjuntamente sobre la materia:

- Cursos en español:

- Administración electrónica.

- Administración electrónica y gobierno digital.

- Alfabetización digital y gestión del patrimonio bibliográfico .

- Archivos: derecho de acceso.

- Aspectos institucionales de la administración electrónica.

- Aspectos penales de la actividad bibliotecaria y archivística.

- Certificados digitales y firma electrónica: una nueva forma de identificarse en internet y con las administraciones.

- Cibercriminalidad.

- Contratación a distancia: comercio electrónico.

- Derecho y gobernanza.

- Documentos jurídicos y estándares.

- Ética y legislación para ingenieros.

- Filosofía del derecho.

- Firma electrónica.

- Gobierno electrónico.

- Las bibliotecas: derecho de acceso versus propiedad intelectual.

- Marco jurídico y deontológico de la actividad documental.

- Observatorios de la sociedad de la información.

- Oficinas de notarios y registradores: documentos y derecho de acceso.

- Ordenadores, móviles e Internet.

- Cursos en inglés:

- Company, law and management.

- Computer law in an international perspective.

- Computers and law.

- Copy right law.

- Cybercrime.

- Data protection.

- E-governance and egovernment.

- E-government activities for environmental information.

- Electronic government.

- Forensics computing.

- Foundations of private law.

- ICT (Information and Communication Technologies) in legal domain.

- Information law.

- Information security law. 
- IT (Information Technologies) law.

- Jurimetrics.

- Law in information society.

- Legal aspects of electronic comerse.

- Legal informatics.

- Legal knowledge management.

- Special topics in legal informatics.

- Curso en alemán:

- Gewerblicher Rechtsschutz.

- Cursos en portugués:

- Gobernanza y constitución.

- Informática jurídica.

- Propriedade intelectual na sociedade da informação.

Las materias señaladas se ofertan agrupadas por especialidades o títulos docentes. Las adoptadas hasta este momento, que son ofrecidas como docencia de postgrado o formación permanente, son, en concreto $^{28}$ :

1. Máster (60 ECTS) en Marco Jurídico para la Sociedad de la Información, cuyas asignaturas obligatorias se denominan: ordenadores, móviles e internet, documentos jurídicos y estándares y derecho y gobernanza.

2. Diplomas de especialización (30 ECTS) en: oficina judicial, otras oficinas jurídicas (abogados, notarios, procuradores [...], administración y gobierno electrónicos, gobernanza, comercio electrónico: marco jurídico y ética y legislación para ingenieros.

3. Además las asignaturas que integran el Master y los Diplomas pueden realizarse en forma individual (6 ECTS).

A partir del curso 2008-9 se ofrecen contenidos docentes que integran enseñanza oficial dirigida a la obtención de titulaciones de grado y de postgrado referidas a derecho, gestión empresarial, documentación o ingenierías, ofertados desde Centros situados en los varios países en los que se sitúan los grupos de investigación y docencia que participan en las iniciativas docentes constituidas dentro de lo que se llama el Campus Virtual Compartido en Derecho y Tecnologías de la Información y la Comunicación. 


\subsection{El Campus Virtual Compartido en Derecho y Tecnologías de la Información y la Comunicación}

En la actualidad estos contenidos se desarrollan y ponen en práctica en el campo de acción que proporciona un campus virtual, promovido por la Unión Europea, que tiene por objetivo la implantación de estudios sobre derecho y TIC que se puedan realizar a distancia por parte de estudiantes matriculados en las universidades que integran el campus ${ }^{29}$.

En el campus se ofertan, por ahora, los módulos, titulaciones y materias señaladas en el apartado anterior que, explicadas de una forma tradicional, utilizando el recurso a las lecciones magistrales y ejercicios prácticos, también son completadas por contenidos que están expuestos o son accesibles en internet atendiendo a necesidades expresadas por alumnos concretos, identificados mediante el uso por los mismos de las técnicas de cifrado, en concreto de claves públicas.

Dichas asignaturas se explican también, utilizando módulos adaptados, a estudiantes que tan solo utilizan como recursos de aprendizaje los que se facilita por internet. La expansión de este tipo de estudios en enseñanzas oficiales a medio plazo es el objetivo del Campus.

Especialidades y asignaturas se sirven como herramientas auxiliares de los contenidos de documentos jurídicos: manuales, trabajos académicos, informes, textos legales, sentencias y doctrina, propuestos por el profesor en colaboración con los alumnos, atendiendo a las denominaciones de los estudios, módulos, títulos, diplomas y asignaturas en las que se articula la oferta de aprendizaje.

También se utiliza como referencia los materiales que los propios alumnos elaboran mediante su estudio y trabajo expresado en forma de Wikipedia a partir de los módulos que forman parte de la red virtual ${ }^{30}$.

El material es de libre acceso a los alumnos matriculados como también lo es el material propuesto por el profesor.

El material o los documentos se recuperan utilizando palabras contenidas en los mismos que, a modo de tesauros, sirven como palabras de búsqueda ${ }^{31}$.

Las colecciones de palabras seleccionadas por profesores constituyen tesauros organizados, expresados en el idioma propio de quienes los construyen. 
Más en concreto, está en fase de elaboración un sistema que permitirá que la recuperación del material de aprendizaje se produzca utilizando los siguientes mecanismos:

1. Palabras contenidas literalmente en los documentos o materiales a recuperar.

2. Palabras claves seleccionadas por profesores expertos sobre las materias o documentación almacenadas, y;

3. Esquemas conceptuales del material, desarrollados por documentalistas utilizando clasificaciones o esquemas estándares de conocimiento.

Serán considerados como elementos integrantes del material: los seis volúmenes de la serie LEFIS (más de 44 trabajos, expuestos en inglés, español y portugués $)^{32}$, otros escritos, documentos LEFIS, legislación y jurisprudencia. La legislación, reglamentos y jurisprudencia estarán almacenados en sistemas de libre acceso.

Las herramientas indicadas son el punto de partida para la construcción de una Wikipedia/Enciclopedia, cuyo uso estará abierto en internet a las consultas hechas por todo tipo de anónimos, no identificados, navegantes interesados.

Las herramientas que, en cambio, permitan acceso individualizado a determinada información garantizarán su libertad, asegurando el derecho del usuario a la protección de datos personales y a la libertad de consulta, así como la salvaguardia de su confianza personal, pudiendo utilizar para ello entidades de certificación de clave pública que hayan sido elegidas por los usuarios.

\subsection{La red LEFIS}

Varias instituciones de enseñanza superior, europeas y americanas preferentemente $^{33}$, trabajan, prácticamente desde la segunda mitad de los años 90, en la elaboración conjunta de titulaciones, módulos docentes, asignaturas, actividades formativas, proyectos de investigación, desarrollo e innovación, conferencias y materiales auxiliares, dirigidos a la implantación de un aprendizaje del derecho acomodado a las necesidades presentes de lo que se viene a denominar la sociedad del conocimiento, y que hoy (desde 1998-9) plasma en la reforma del Espacio Europeo de Enseñanza Superior (EEES) ${ }^{34}$.

Las experiencias realizadas permiten contar con las anteriormente expresadas asignaturas y contenido de grados y postgrados, de ámbito jurídico-social y técnico, que han sido y son impartidos tanto en universidades que han 
acomodado su práctica docente a las exigencias de la reforma de Bolonia, como en universidades que todavía no lo han hecho pero que lo harán en un plazo más o menos corto ${ }^{35}$.

Las actividades mencionadas son las propias de la red temática LEFIS: Legal Framework for the Information Society (www.lefis.org), que está integrada por 126 instituciones dedicadas a impartir formación superior, preferentemente, interesadas en participar en las iniciativas conjuntas que se articulan en los diferentes formatos que mencionábamos al comienzo de este apartado ${ }^{36}$.

Las instituciones están situadas en 44 países.

El campus virtual mencionado con anterioridad constituye las actividades desenvueltas desde Octubre de 2007 por 11 universidades, 10 europeas y 1 sudamericana (situada en Brasil). Iniciativa que es posible poner en práctica en la actualidad gracias a la progresiva implantación institucional del EEES $\mathrm{y}$ a los recursos que proporcionan los nuevos métodos de aprendizaje ${ }^{37}$, es decir: los medios que ponen a disposición del profesorado, estudiantes y técnicos participantes, 1) los recursos multimedia y la enseñanza a distancia, 2) la globalización de las actividades sociales y 3) las posibilidades que a su dinamización les aporta el desarrollo tecnológico, como señala la Declaración de Lovaina de Abril de 2009 en su Preámbulo, última Declaración relevante del proceso de reforma del EEES.

Se especifica a continuación las universidades que participan en el Campus Virtual Law\&ICT indicando el país y el lugar en el que están domiciliadas y el nombre de la institución: Alemania (Münster), España (La Laguna y Zaragoza), Finlandia (Vaasa y Laponia: Rovaniemi), Lituania (Mikolas Romeris: Vilnius), Polonia (Nicolás Copérnico: Torun), Portugal (Instituto Politécnico de Beja), Reino Unido (Queen's: Belfast) y Turquía (Bahcesehir: Estambul). Constituye, también, parte de la oferta docente del grupo la que se realiza, en coordinación con las ofertas de las restantes universidades, por la Universidad Federal de Santa Catalina (Florianópolis, Brasil).

\section{CONCLUSIÓN}

El trabajo ha puesto de relieve que sin atender al que es el contexto de cada momento, o lo que es lo mismo, el campo de acción propio de los juristas, las mismas actividades jurídicas, y las funciones sociales de las instituciones en donde se realizan dichas actividades, no es posible realizar investigaciones de carácter jurídico. 
También ha podido observarse que la toma en consideración aislada del producto técnico que aportan los conocimientos técnico-científicos, e, incluso, de las hipotéticas derivaciones del contenido abstracto de las propias normas desarrolladas por la teoría del derecho o los sistemas expertos, son aproximaciones limitadas, que no permiten evidenciar la relevancia de los desarrollos, aplicaciones o críticas realizadas ni, lo que aquí es más importante, incrementar las virtualidades del propio desarrollo tecnológico.

El desarrollo tecnológico solo se ve realmente potenciado cuando se contempla y usa sus virtualidades mediante la elaboración de aplicaciones, realizadas por grupos de investigación, que están en sintonía con el campo de acción propio de actividades jurídicas realizadas en un ámbito jurídico democrático.

1 El desarrollo tecnológico es uno de los causantes de la sociedad en red o del derecho actual, pero no el único. Por supuesto otro es el fenómeno de la globalización, caben otros más: el de la multiculturalidad, por ejemplo. Aquí no entramos ni en la reseña ni en el estudio de esos otros motivos, lo que no impide reconocer que siempre está presente en el trabajo su relevancia

2 La referencia básica desde el comienzo de las investigaciones ha sido el trabajo de Jon Bing sobre acceso a documentación jurídica (Bing, 1984).

3 Un informe de relevancia sobre el tema se encuentra en: Dumortier , J., Kelm, S., Nilsson, H., Skouma, G. \& Vas Eecke, P. The Legal and Market Aspects of Electronic Signatures in Europe. Study for the European Commission within the e Europe 2005 framework, Leuven, Interdisciplinary Centre for Law and ICT, 2005. El informe está accesible en: http://ec.europa.eu/information_society/eeurope/2005/all_about/security/ electronic_sig_report.pdf (Consultado el 18 de agosto de 2009).

4 El grupo fue reconocido como grupo de investigación por el Gobierno de Aragón en 2003. Recibe financiación anual de este Gobierno en forma complementaria a la financiación obtenida en concursos públicos y mediante la realización de proyectos de I+D. El grupo está integrado por 16 miembros que trabajan en la Universidad de Zaragoza, la Universidad de San Jorge y la Diputación Provincial de Zaragoza. Los miembros del grupo trabajan en Derecho, Matemáticas, Documentación e Informática. Hay información sobre las actividades desarrolladas por el grupo en: http://www.lefis.org/index.php?option=com_content\&task=view\&id=173\&It emid=511 (Consultado el 18 de agosto de 2009)

5 www.lefis.org. LEFIS es una marca europea registrada. En concreto tiene el número 005625132. El registro está publicado el 10/12/2007 en el Bolletin of comunitarian marks número 2007/066.

6 Galindo, F., «El derecho, ¿habla o lenguaje? Una aproximación a su carácter a través de la informatización de una disposición jurídica». En: Martin Vide, C., (ed.). Lenguajes Naturales y Lenguajes Formales. Barcelona: Facultad de Filología, 1985.

7 Ver: http://www.unizar.es/derecho/standum_est_chartae/ (Consultado el 18 de agosto de 2009).

8 Otro ejemplo que mostró las posibilidades limitadas del uso de estos recursos en derecho fue el sistema experto sobre el Latent Damage Act de1986 (Capper, Susskind, 1988).

9 Galindo, F. «Expert Systems as Tools for the Explanation of the Legal Domain: The ARPO Experiences». En: Expert Systems with Applications. An International Journal, vol. 4, 1992.

${ }_{10}$ Galindo, F. «The legal decision as a political problem. A starting point for a profitable relationship between $\mathrm{AI}$ and Law». En: Adversarial Reasoning and Artificial Intelligence and Legal Reasoning. Workshop Notes from the Eight National Conference on Artificial Intelligence (AAAI-90), Boston, 1990.

11 Sobre el concepto comunicativo de derecho, ver también: Galindo, F. «The communicative concept of law». Journal of legal pluralism and unofficial law. Vol. 41, 1998.

12 Baaz, M., Galindo, F., Quirchmayr, G., Vázquez, M. «The appplication of Kripke- Type structures to regional development programs». En: Marik, V., Lazansky, J., Wagner, R., (eds.). Database and Expert Systems Applications. Springer verlag. Heidelberg, 1993.

13 Pastor, J., Delgado, J., Galindo, F., (eds.). Criptografía, Privacidad y Autodeterminación informática. Zaragoza: Facultad de Derecho y Centro Politécnico Superior, 1995. 
${ }_{14}$ Este es el movimiento "Law via the Internet". Las conferencias realizadas desde 1997 están situadas en: http://www.ittig.cnr.it/LawViaTheInternet/index.php?option=com_content\&view=article\&id=16\&Itemid=6 1 (Consultado el 18 de agosto de 2009) .

15 Se pueden localizar referencias sobre esta evolución en España en: http://www.poderjudicial.es/eversuite/GetDoc?DBName=dPortal\&UniqueKeyValue=\%2025039\&Download $=$ false\&ShowPath=false (Consultado el 18 de agosto de 2009).

${ }^{16}$ Un temprano y concreto ejemplo en relación con las actividades de la Administración Pública e Internet están en: Galindo, F., Muñoz, J.F., Rivas, A. «Elaboración de un plan estratégico para la implantación del correo electrónico en la Diputación General de Aragón». En: IV Jornadas sobre las tecnologías de la información para la modernización de las Administraciones Públicas. TECNIMAP'95. Ministerio para las Administraciones Públicas. Madrid, 1995.

17 Galindo, F., Quirchmayr, G., (eds.). Advances in Electronic Government. Pre-Proceedings of the Working Conference of the International Federation of Information Processing, Zaragoza, Seminario de Informática y Derecho. Universidad de Zaragoza, 2000.

${ }^{18}$ Las Directivas básicas fueron: Directive 95/46/EC of the European Parliament and of the Council of 24 October 1995 on the protection of individuals with regard to the processing of personal data and on the free movement of such data, Directive 1999/93/EC of the European Parliament and of the Council of 13 December 1999 on a Community framework for electronic signatures, Directive 2000/31/EC of the European Parliament and of the Council of 8 June 2000 on certain legal aspects of information society services, in particular electronic commerce, in the Internal Market ('Directive on electronic commerce') y la Directive 2002/58/EC of the European Parliament and of the Council of 12 July 2002 concerning the processing of personal data and the protection of privacy in the electronic communications sector ('Directive on privacy and electronic communications').

19 El proyecto más significativo fué el apoyado por la Unión Europea denominado AEQUITAS: The Admission as Evidence in Trials of Penal Character of Electronic Products Signed Digitally. El informe final (1998) está situado en: http://cordis.europa.eu/infosec/src/study11.htm (Consultado el 18 de agosto de 2009).

${ }^{20}$ Galindo, F. «Public Key Certification Providers and E-government Assurance Agencies. An Appraisal of Trust on the Internet». En: Database and Expert Systems Applications. Workshops. Los Alamitos. IEEE, 2001.

${ }^{21}$ Las actividades realizadas conjuntamente con los Notarios españoles estuvo en el origen de la Agencia Notarial de Certificación http://www.ancert.com (Consultado el 18 de agosto de 2009).

22 Ver la infraestructura de clave pública LEFIS UNIZAR en: https://lefis.unizar.es/pki/index.php?lang=es (Consultado el 18 de agosto de 2009).

23 "Sociedad omnipresente" o "Ubiquitous society" es una expresión habitual en los países del Norte de Europa: Aarnio, R. «Data protection here and now». En: Saarenpää, A., (ed.) Legal privacy. LEFIS Series. Vol. 5. Zaragoza: Prensas Universitarias, 2008.

${ }^{24}$ Galindo, F. «Justicia, gobernanza y legalidad». En: Seqüência. Vol. 17, 2007.

25 Galindo, F. (ed.). Gobierno, Derecho y Tecnologías: las actividades de los poderes públicos. Cizur Menor., Thomson Civitas, 2006.

${ }^{26}$ Galindo, F. (ed.). «LIACTES/IFIP Workshop on E-Government: Legal, Technical and Pedagogical Aspects, Albarracin Spain, 8-10 May 2003». The Journal of Information, Law and Technology (JILT). http://www2.warwick. ac.uk/fac/soc/law/elj/jilt/2003_1/liactes (Consultado el 18 de agosto de 2009).

27 El Campus Virtual Compartido sobre Derecho y TIC: Law\&ICT Shared Virtual Campus, está apoyado por la Unión Europea, Programa Capus Virtuales Proyecto 133837-LLP-1-2007- ES-ERASMUS-EVC. La página web está situada en: http://www.lawict.eu/ (Consultado el 18 de agosto de 2009).

${ }^{28}$ Ver más detalles en: http://courses.lefis.unizar.es/index.php?option=com_content\&task=view\&id=94\&Itemi $\mathrm{d}=241$ (Consultado el 18 de agosto de 2009) .

29 Ver supra nota 28.

30 Hablamos de LEFISpedia. Una aproximación inicial está situada en: http://www.lefis.org/wiki/index.php5/ Conceptos (Consultado el 18 de agosto de 2009).

31 Hablamos de la Biblioteca digital LEFIS. Una aproximación inicial está situada en: http://courses.lefis.unizar. es/index.php?option=com_wrapper\&Itemid=335 (Consultado el 18 de agosto de 2009)

32 La colección está situada en: http://puz.unizar.es/catalogo/colecciones_libros.php?coleccion=40 (Consultado el 18 de agosto de 2009).

33 También hay instituciones radicadas en India, China y Rusia, por ejemplo.

${ }_{34}$ Sobre el proceso de reforma ver, en general: Michavila 2008, en Derecho: Galindo 2009.

35 La heterogeneidad de aproximaciones y puestas en práctica por países del proceso de Bolonia puede verse en: Ederer y otros 2008. 
Fernando Galindo - Derecho e internet

36 Ver el Informe final de LEFIS en: http://www.lefis.org/images/documents/management/Progress_and_final_ reports/lefis/Informe_Final_Lefis_2007_EN-DEFGALk.pdf (Consultado el 18 de agosto de 2009).

37 Una valoración, con ejemplos, de las posibilidades del uso los medios telemáticos en la docencia se encuentra en: Moral y otro 2009. 